\title{
Stage 0 Anal Cancer AJCC v8
}

National Cancer Institute

\section{Source}

National Cancer Institute. Stage O Anal Cancer A/CC v8. NCI Thesaurus. Code C133795.

Stage 0 includes: Tis, NO, MO. T is: High-grade squamous intraepithelial lesion (previously termed carcinoma in situ, Bowen disease, anal intraepithelial neoplasia II-III, high-grade anal intraepithelial neoplasia). N0: No regional lymph node metastasis. M0: No distant metastasis. (AJCC 8th ed.) 